\title{
A Classic Type 2 QSO
}

Colin Norman ${ }^{1,2}$, Guenther Hasinger ${ }^{3,4}$, Riccardo Giacconi ${ }^{1,5}$, Roberto Gilli ${ }^{1,6}$, Lisa Kewley $^{1,7}$, Mario Nonino ${ }^{8}$, Piero Rosati ${ }^{1,9}$, Gyula Szokoly ${ }^{3}$, Paolo Tozzi ${ }^{8}$, Junxian Wang ${ }^{1}$, Wei Zheng ${ }^{1}$, Andrew Zirm ${ }^{1}$, Jacqueline Bergeron ${ }^{9}$, Roberto Gilmozzi ${ }^{9}$, Norman Grogin ${ }^{2}$, Anton Koekemoer ${ }^{2}$, and Ethan Schreier ${ }^{2}$

\begin{abstract}
In the Chandra Deep Field South 1Msec exposure we have found, at redshift $3.700 \pm 0.005$, the most distant Type 2 AGN ever detected. It is the source with the hardest X-ray spectrum with redshift $z>3$. The optical spectrum has no detected continuum emission to a $3 \sigma$ detection limit of $\sim 3 \times 10^{-19} \mathrm{ergs} / \mathrm{s} / \mathrm{cm}^{2} / \AA$ and shows narrow lines of Ly $\alpha, \mathrm{CIV}, \mathrm{NV}$, HeII, OVI, [OIII], and CIII]. Their FWHM line widths have a range of $\sim 700-2300 \mathrm{~km} \mathrm{~s}^{-1}$ with an average of approximately $\sim 1500 \mathrm{~km} \mathrm{~s}^{-1}$. The emitting gas is metal rich $\left(Z \simeq 2.5-3 Z_{\odot}\right)$.

In the X-ray spectrum of 130 counts in the $0.5-7 \mathrm{keV}$ band there is evidence for intrinsic absorption with $N_{H} \gtrsim 10^{24} \mathrm{~cm}^{-2}$. An iron $\mathrm{K} \alpha$ line with rest frame energy and equivalent width of $\sim 6.4 \mathrm{keV}$ and $\sim 1 \mathrm{keV}$, respectively, in agreement with the obscuration scenario, is detected at a $2 \sigma$ level. If confirmed by our forthcoming XMM observations this would be the highest redshift detection of FeK $\alpha$. Depending on the assumed cosmology and the X-ray transfer model, the $2-10 \mathrm{keV}$ rest frame luminosity corrected for absorption is $\sim 10^{45 \pm 0.5} \mathrm{ergs} \mathrm{s}^{-1}$, which makes our source a classic example of the long sought Type 2 QSOs. From standard population synthesis models, these sources are expected to account for a relevant fraction of the black-hole-powered QSO distribution at high redshift.
\end{abstract}

${ }^{1}$ The Johns Hopkins University, Homewood Campus, Baltimore, MD 21218

${ }^{2}$ Space Telescope Science Institute, 3700 San Martin Drive, Baltimore, MD 21218

${ }^{3}$ Astrophysikalisches Institute Potsdam, An der Sternwarte 16, Potsdam, D-14482, Germany

${ }^{4}$ Max-Planck-Institut für Extraterrestrische Physik, Giessenbachstrasse, Garching, D-85740, Germany

${ }^{5}$ Associated Universities, Inc. 1400 16th Street, NW, Suite 730, Washington, DC 20036

${ }^{6}$ Osservatorio Astrofisico di Arcetri, Largo E. Fermi 5, 50125 Firenze, Italy

${ }^{7}$ Harvard-Smithsonian Center for Astrophysics, 60 Garden Street, Cambridge, MA 02138

${ }^{8}$ Osservatorio Astronomico, Via G. Tiepolo 11, 34131 Trieste, Italy

${ }^{9}$ European Southern Observatory, Karl-Schwarzschild-Strasse 2, Garching, D-85748, Germany 


\section{Introduction}

The unified model for AGNs is widely accepted. Briefly, the physics of black hole, accretion disk, jet, and obscuring torus is convolved with the geometry of the viewing angle and can explain most of the apparent disparate properties of active galaxies (Antonucci 1993). The use of the word torus here is generic for the obscuring region since there are many variants of the geometry of the obscuring region including a strict toroidal geometry and flaring disk models (Efstathiou \& Rowan-Robinson 1995; Granato et al. 1997). Type 1 objects exhibit the straight physics of AGNs with no absorption and Type 2 objects arise when the view is obscured by the torus. A crucial component that has long been sought are heavily obscured powerful quasars called QSO 2s. They have been predicted to have narrow permitted lines, powerful hard X-ray emission and a high equivalent width Fe $\mathrm{K} \alpha$ line (Ghisellini et al. 1994).

In the absence of a good standard case doubt has been expressed, at times, about the whole QSO 2 phenomenon (Halpern et al. 1999). The successful finding of an optically obscured central AGN in the starburst NGC 6240 (Vignati et al. 1999) made X-rays the obvious wavelength to uncover the Type 2 QSO phenomenon. There have been few previous studies of candidate Type 2 objects in the X-ray band. One has been observed by ROSAT and ASCA (Almaini et al. 1995; Georgantopoulos et al. 1999). Two are underluminous objects from ASCA (Ohta et al. 1996) and Chandra (Fabian et al. 2000). Also, the hyperluminous infrared galaxy IRAS $09104+4109$, observed with BeppoSAX (Franceschini et al. 2000) and recently with Chandra (Iwasawa et al. 2001), appears to be an example of a Type 2 QSO at moderate redshift, $z=0.442$. It is a $\mathrm{cD}$ galaxy in a rich cluster and is radio loud with a radio jet. A recent candidate at high redshift $(z=3.288)$ has been found in the deep Chandra exposure of the Lynx field (Stern et al. 2001).

In parallel with the X-ray work, imaging and spectropolarimetric studies on warm ultraluminous, infrared galaxies (ULIRGs) indicate that all of them might contain buried QSOs (Tran et al. 2000). In addition, high redshift radio galaxies (HZRGs) harbour obscured QSOs, and intensive studies are underway to understand the physics of the central regions of these objects (Vernet et al. 2001).

From our multiwaveband studies of the 1Msec exposure of the Chandra Deep Field South (CDF-S) (Giacconi et al. 2001a; Tozzi et al. 2001; Rosati et al. 2001; Giacconi et al. 2001b) we now discuss a classic X-ray selected Type 2 QSO: CXOCDFS J033229.9 -275106 (hereafter CDF-S 202). We note in passing that, in contrast to the above mentioned radio studies, CDF-S 202 is radio quiet.

The Paper is organized as follows. In Section II we describe the multiwavelength obser- 
vations we use. In Section III we present and analyze the optical spectrum. In Section IV we discuss the X-ray spectrum. In Section V we study the object further and present some of the wider implications of this work. We conclude and consider future work in the final section.

We assume a cosmology in this paper with the parameters $H_{0}=60 \mathrm{~km} \mathrm{~s}^{-1} \mathrm{Mpc}^{-1}$, $\Omega_{m}=0.3$ and $\Omega_{\Lambda}=0.7$.

\section{Imaging Observations}

We have combined 11 individual Chandra pointings into a mosaic with a maximum of $942 \mathrm{ksec}$ of exposure time (hereafter the $1 \mathrm{Msec}$ exposure). The limiting fluxes achieved are $5.5 \times 10^{-17} \mathrm{erg} \mathrm{cm}^{-2} \mathrm{~s}^{-1}$ in the $0.5-2 \mathrm{keV}$ band and $4.5 \times 10^{-16} \mathrm{erg} \mathrm{cm}^{-2} \mathrm{~s}^{-1}$ in the 2-10 $\mathrm{keV}$ band (Rosati et al. 2001). The details of the X-ray observations are given in Giacconi et al. (2001b, submitted). Among the sources with $z>3$, CDF-S 202 is the one with the hardest X-ray spectrum. In the X-ray image from $0.5-7 \mathrm{keV}$ the source is $\sim 3^{\prime}$ from the center, where the point response function has a full-width-half-maximum (FWHM) of $1^{\prime \prime}$. The X-ray intensity profile is completely consistent with that of a point-source at this resolution. There are no other X-ray sources detected within $30^{\prime \prime}$.

The primary optical ID was made in deep $R$ and $I$ band images $(R($ Vega $) \leq 26)$ obtained using the Focal Reducer Spectrograph (FORS1) on the European Southern Observatory Very Large Telescope $8.2 \mathrm{~m}$ facility (ESO/VLT-ANTU). All magnitudes quoted in this paper are Vega magnitudes. CDF-S 202 has an $R$ magnitude of 23.53 and an $I$ magnitude of 22.65 . We have additional photometric coverage of this object in the $U, V, B, J$ and $K s$ bands. The $\mathrm{B}$ band used here is from ESO Wide-Field Imager data taken on the $2.2 \mathrm{~m}$ ESO telescope, which reaches a limiting magnitude of 26 . The near-infrared data comes from the ESO Imaging Survey (EIS) and reaches 23.6 in $J$ and 21.8 in $K s$. Of these five bands our candidate QSO 2 is detected significantly in $V$ and $K s$, at 25.27 and 20.99 respectively. The $B$-band source is very faint, essentially at the limiting magnitude. The images in the $B, R, I, J$ and $K s$ bands are given in Fig. 1. 


\section{Optical Spectroscopy}

\subsection{Observations and Data Reduction}

The optical spectrum was obtained with the multislit mode on the Focal Reducer Spectrograph (FORS1) on the European Southern Observatory Very Large Telescope 8.2m unit facility (ESO/VLT-ANTU) on 11-25-2000. The FORS instrument is described in detail in Mitsch et al. (1994). The spectroscopy was obtained using the $150 \mathrm{I}+17$ grism and no order separation filter, which allows maximum wavelength coverage but introduces second order contamination. This grism gives a spectral resolution of $5.5 \AA /$ pixel. The detector slit width was 1.2 arcseconds, (around $30 \AA$ dispersed), chosen to maximize the signal-to-noise ratio of the resulting spectra (i.e. including most of the light from the object, but minimizing the sky background). The final spectrum is composed of 7 exposures with a total integration time of $\sim 3$ hours.

Data reduction was carried out using the IRAF package. This involved bias subtraction, flat fielding, background subtraction, wavelength and flux calibration. The background was subtracted by fitting a second order polynomial to each column. The spectrum was extracted using an aperture width of 14 pixels, which was sufficent to include the total emission from the object.

Wavelength calibration was derived using the spectra from four arc lamps ( $\mathrm{He}, \mathrm{HgCd}$ and two separate Ar lamps) taken on the same night. Line centers and shapes can only be determined to a few $\AA$ accuracy due to the finite slit width. In addition, the exact location of the object on the slit can introduce an additional few $\AA$ systematic shift in the spectrum. These limit the redshift to an accuracy of \pm 0.005 . It is also important to note that the finite slit size and seeing introduces an artificial broadening of the lines. As the seeing is potentially wavelength dependent, this effect can be also wavelength dependent. We estimate this effect to be a few times $10 \AA$. Therefore, the line widths measured (see Table 1) should be considered upper limits.

The spectra were flux calibrated using very wide (5 arcsec) slits using both a red and a blue photometric standard star for comparison. As the seeing is potentially wavelength dependent, applying this flux calibration can introduce a wavelength dependent slit-loss (in addition to the varying slit loss introduced by the atmospheric refraction). Considering the relatively good seeing (0.5" FWHM at the beginning of the observation) and low airmass, this

color effect is not very strong. Second order contamination was removed from the specphot standard observations using two standards with very different spectral shape. This made it possible to calibrate the true throughput of the system. Due to the very faint continuum of CDF-S 202, the second order contamination was not removed from its spectra. This implies 
that the continuum shape would be incorrect (if we could detect it), but the emission line features are correctly calibrated. Comparing the spectra obtained using each photometric standard, we estimate that our flux calibration is accurate to 5\%. At present, we use known CTIO extinction curves until ESO extinction curves for Paranal become available to us.

\subsection{Emission-Line Analysis}

QSOs can be classified into Type 2 and Type 1 objects in a similar fashion to Seyfert galaxies, which are generally classified based on their relative emission-line widths using the scheme proposed by Weedman $(1970,1973)$ and Khachikian \& Weedman $(1971,1974)$. In this scheme, Seyferts which show broad H I, He I and He II emission lines FWHM $\sim 3-5 \times 10^{3} \mathrm{~km} \mathrm{~s}^{-1}$ ) are known as Seyfert 1 galaxies. These galaxies also display strong blue continua, and frequently complexes of broad Fe II emission are also seen. Superimposed on this spectrum are 'narrow' forbidden lines, which are thought to be formed in a much larger extended narrow line region (NLR) around the nucleus.

The forbidden lines in Seyfert 1 galaxies such as [O III], [N II], and [S II] typically have FWHM of $\sim 5 \times 10^{2} \mathrm{~km} \mathrm{~s}^{-1}$. Galaxies with permitted and forbidden lines with approximately the same FWHM (typically $\sim 5 \times 10^{2} \mathrm{~km} \mathrm{~s}^{-1}$ ) are called Seyfert 2 galaxies. The broad lines are absent in such objects and they display a flat, featureless continuum (eg., Kinney et al. 1993; Heckman et al. 1995). Similarly, broad QSO emission lines typically have widths of $3000-5000 \mathrm{~km} \mathrm{~s}^{-1}$, while narrow QSO emission lines are a few hundreds of $\mathrm{km} \mathrm{s}^{-1}$ (eg., Peterson 1997; Forster et al. 2001).

To determine the emission line fluxes and FWHM for CDF-S 202, the optical spectral line profiles were modeled by one Gaussian per line using the ngaussfits task in IRAF. This routine uses least squares fitting implemented by a downhill simplex minimization algorithm. The resulting emission line fluxes and FWHM found for the emission lines in CDF-S 202 are given in Table 1. The final spectrum is shown in Figure 2. The permitted emission lines, $\mathrm{O}$ IV, Ly $\alpha, \mathrm{N}$ V, $\mathrm{C}$ IV, and He II have $\mathrm{S} / \mathrm{N}$ ratios $>3 \sigma$ and are well identified, giving a redshift of $z=3.700 \pm 0.005$. The continuum is not detected to a $3 \sigma$ limit of $\sim 3 \times 10^{-19}$ $\operatorname{ergs} / \mathrm{s} / \mathrm{cm}^{2} / \AA$. This is consistent with the flat, featureless continua seen in many Type 2 objects such as NGC 3393 (eg. Kinney et al. 1993; Heckman et al. 1995).

The emission line widths of CDF-S 202 are given in Table 1. They have a rest-frame FWHM of between $\sim 700-2300 \mathrm{~km} \mathrm{~s}^{-1}$. Note that these FWHM are actually upper limits

as discussed in the previous section, and that the "true" FWHM may be even less than those quoted here. The FWHM of Ly $\alpha$ has an upper limit of $1100 \mathrm{~km} \mathrm{~s}^{-1}$. Assuming that 
our FWHM upper limits are close to the true FWHM of the emission lines (within 100-300 $\mathrm{km} \mathrm{s}^{-1}$ ), then our Ly $\alpha$ FWHM is similar to the FWHM of $\sim 900 \mathrm{~km} \mathrm{~s}^{-1}$ observed in the Type 2 QSO IRAS 09104+4109 (Kleinmann et al. 1988). Such FWHM are broader than those seen in typical Seyfert $2 \mathrm{~s}\left(\sim 5 \times 10^{2} \mathrm{~km} \mathrm{~s}^{-1}\right)$, but narrower than those observed in Seyfert $1 \mathrm{~s}$ and broad-line QSOs $\left(3000-5000 \mathrm{~km} \mathrm{~s}^{-1}\right)$. Our FWHM are, however, similar to those found in the so-called "narrow-line Seyfert 1" galaxies which have FWHM of 1000-2000 $\mathrm{km} \mathrm{s}^{-1}$ (Goodrich 1989). However, narrow-line Seyfert 1 galaxies are generally defined as having Balmer lines slightly broader than the forbidden lines, an [O III $] \lambda 5007 / \mathrm{H} \beta$ ratio less than 3, and in most cases, the presence of high ionization iron species. Without spectra at optical wavelengths in the rest-frame, and better estimates of the FWHM, we are unable to determine whether CDF-S 202 fits these criteria.

We note that the absorption seen in CDF-S 202 of $N_{H} \gtrsim 10^{24} \mathrm{~cm}^{-2}$ is common for Type 2 objects (Maiolino et al. 1998; Risaliti, Maiolino \& Salvati 1999) but not for Type 1s (Reeves \& Turner 2000). In addition, the flat, featureless continuum to a $3 \sigma$ flux limit of $\sim 3 \times 10^{-19} \mathrm{ergs} / \mathrm{s} / \mathrm{cm}^{2}$ supports a Type 2 classification of CDF-S 202.

Figure 3 shows the $\mathrm{N} \mathrm{V} / \mathrm{He}$ II vs. $\mathrm{N}$ V/C IV diagram with models by Vernet et al. (2001) and Hamann \& Ferland (1993). Hamann \& Ferland (1993) showed that high redshift quasars $(z>2)$ define a tight correlation, indicated by the dashed line in this figure. This correlation is predominantly a result of metallicity variations in which the higher redshift quasars have higher metallicities. This redshift-metallicity effect may be due to higher QSO or host galaxy masses at higher redshifts. From Figure 3, we can see that the N V/C IV and N V/He II ratios for CDF-S 202 are strong, similar to those previously seen in Type 1 QSOs and powerful radio galaxies at similar redshifts (Hamann \& Ferland 1993; Vernet et al. 2001). The position of CDF-S 202 in this figure indicates emission line ratios intermediate between HzRGs and BLR QSOs.

The solid line shown in Figure 3 gives the best-fit power-law photoionization model for high redshift radio galaxies $(\mathrm{HzRG})$ run by Vernet et al. for metallicities from $0.4-4 Z_{\odot}$. Similarly, the Hamann \& Ferland (1993) model for the broad emission-line region of QSOs (QSO BLR) is shown in dashes. From these models, CDF-S 202 appears to be a high metallicity $\left(\mathrm{Z} \sim 2.5-3 \times Z_{\odot}\right)$ object, at the high end of the metallicity range found in HzRGs by Vernet et al. although an independent metallicity diagnostic is required to verify this.

The He II/C IV ratio is $\sim 0.3$. Heckman et al. (1995) note that this ratio is typically 0.1 in Seyfert 1 galaxies compared with 0.9 for Seyfert 2 galaxies. Narrow-line radio galaxies (the radio-loud analogs to Seyfert 2 galaxies) also show strong He II/C IV ratios $(\sim 0.9)$ (McCarthy 1993), while spectra of high- $z$ quasars resemble the Seyfert 1 galaxies 
with He II/C IV 0.15 (Francis et al. 1991). The He II/C IV ratio for CDF-S 202 is intermediate between the Type 1 and Type 2 values. However, CDF-S 202 is more likely a Type 2 QSO rather than an intermediate QSO, since its emission line velocity widths are relatively narrow and heavy obscuration is confirmed from the $\mathrm{X}$-rays.

\section{X-ray Spectroscopy}

The X-ray spectrum of CDF-S 202 and the relative background spectrum were extracted from the $\mathrm{X}$-ray image using the standard CIAO v2 software. The source spectrum was extracted in a circle with radius $R=5^{\prime \prime}$, the background spectrum was extracted in an annulus between $R+2^{\prime \prime}$ and $R+12^{\prime \prime}$ around the source position. We verified that changing the source extraction radius does not affect significantly our results. With the same software we produced response matrix functions at the CDF-S 202 position. The $\mathrm{X}$-ray spectrum of CDF-S 202 was then analyzed using XSPEC v11.0. The data were rebinned to have at least 10 counts per bin (source + background), in order to validate the use of the $\chi^{2}$ statistics. Hereafter errors are quoted at the $90 \%$ confidence level for one interesting parameter $\left(\Delta \chi^{2}=\right.$ 2.71). The fit with a simple power law absorbed by the Galactic hydrogen gives a very flat slope $(\Gamma=0.51 \pm 0.27)$ suggesting heavy absorption. We then considered two possible scenarios. First, we assumed the $\mathrm{X}$-ray emission is transmitted through an absorber which cuts off the spectrum at low energies. Usually the absorber is identified with the putative obscuring torus expected from unified schemes, although its geometry could be different. Second, we assumed the X-rays are reflected. The obscuring material is then optically thick to Compton scattering with no transmitted X-rays observed in the Chandra band up to $7 \times(1+z) \sim 33 \mathrm{keV}$, implying $N_{\mathrm{H}} \gtrsim 10^{25} \mathrm{~cm}^{-2}$. In this case the X-rays are reflected by the inner edge of the torus (or any cold cloud off the line of sight). Generally, for large equivalent width of the $\mathrm{Fe} \mathrm{K} \alpha$ line, the reflection model is favoured (Ghisellini et al. 1994).

We first considered the transmission model and fitted the spectrum with an absorbed powerlaw. The best fit photon index $(\Gamma=1.847)$ is poorly constrained, therefore we fixed it to be $\Gamma=1.8$, a standard value for AGN, deriving a column density of $N_{\mathrm{H}}=6.7_{-2.1}^{+2.4} \times 10^{23}$ $\mathrm{cm}^{-2}$. A residual excess around $1.4 \mathrm{keV}$ suggested the presence of an iron $\mathrm{K} \alpha$ line at 6.4 $\mathrm{keV}$ rest frame $(6.4 /(1+3.700) \simeq 1.4 \mathrm{keV})$, therefore we added a narrow Gaussian line to the model fixing the line energy to $6.4 \mathrm{keV}$ and the line width to $0 \mathrm{keV}$. The addition of the iron line changes the $\chi^{2} /$ dof value from $11.6 / 16$ to $9.5 / 15$; the fitted column density slightly increases to $N_{\mathrm{H}}=7.9_{-2.3}^{+3.5} \times 10^{23} \mathrm{~cm}^{-2}$. Although the iron line detection is not significant $(<2 \sigma$ level according to an $F$-test for one additional free parameter), we will leave the line in the transmission model for comparison with the reflection model. The line equivalent width 
in the observed frame is $E W_{K \alpha}=175_{-175}^{+360} \mathrm{eV}$, corresponding to $E W_{K \alpha}=823_{-823}^{+1694} \mathrm{eV}$ in the rest frame. The $2-10 \mathrm{keV}$ rest frame luminosity is calculated to be $4.4 \times 10^{43} \mathrm{erg} \mathrm{s}^{-1}$. When correcting for absorption, the intrinsic 2-10 keV rest frame luminosity is $3.3 \times 10^{44} \mathrm{erg} \mathrm{s}^{-1}$. In Figure 4 we show the fit to the $\mathrm{X}$-ray spectrum together with the best fit transmission model.

We then considered a pure reflection model using the pexrav model in XSPEC with $\Gamma$ fixed to 1.8 and adding at $\sim 6.4 \mathrm{keV}$ rest frame a narrow Gaussian line with $\sigma_{K \alpha}=0 \mathrm{keV}$. The description of the data is as good as in the transmission model $\left(\chi^{2} / d o f=10 / 15\right.$; see Figure 5). With this model the line is detected at $>2 \sigma$ level according to an $F$-test for one additional free parameter $\left(\Delta \chi^{2}=4.6\right.$ with respect to the same reflection model without the line). The rest frame line energy and equivalent width are $E_{\mathrm{K} \alpha}=6.43 \pm 0.34 \mathrm{keV}$ and $E W_{\mathrm{K} \alpha}=1186_{-922}^{+1195} \mathrm{eV}$, respectively. The line equivalent width is then in full agreement with the expectations from Compton thick sources (Ghisellini et al. 1994). Assuming a reflection efficiency of 3\% (see e.g. Krolik, Madau \& Zicky 1994; Maiolino et al. 1998; and Sect 3.1 in Gilli, Risaliti \& Salvati 1999), the $2-10 \mathrm{keV}$ rest frame intrinsic luminosity of the source is $1.410^{45} \mathrm{erg} \mathrm{s}^{-110}$.

In principle one could look at the source variability to estimate which scenario is favored. Indeed, short term variability, on timescales of a few $10 \mathrm{ksec}$, indicates that the observed radiation is transmitted rather than reprocessed by a pc-scale reflecting material. Unfortunately, analysis of short term variability is not possible given our photon statistics. Also, long term variability (the data were collected in a 14 months period) is poorly constrained.

We also note in passing the blip in the $\mathrm{X}$ - ray spectrum at $3.2 \mathrm{keV}$, corresponding to a restframe energy $\sim 15 \mathrm{keV}$. This is not a statistically significant line in the spectrum but we point out that such features have found twice previously in ASCA observations of other AGN and may be associated with Doppler-bosted FeK $\alpha$ (Yaqoob et al. 1999).

\section{Discussion and Implications}

How common are QSO 2s and how can more be detected? This object stood out in a Hardness Ratio-Redshift plot of the currently cataloged sources in the Chandra Deep Field South as the highest redshift source with the hardest spectrum. One would expect to observe the red $(R-K s \gtrsim 4)$ colors of the host galaxy (see e.g. Fig. 7 in Lehmann et al. 2001). In this case, at $z=3.700$, the $R-K s$ color of $\sim 2.5$ does not reflect the galactic continuum, but

\footnotetext{
${ }^{10}$ We note that assuming $H_{0}=50 \mathrm{~km} / \mathrm{s} / \mathrm{Mpc}$ and $q_{0}=0$ gives luminosities higher by a factor of $\sim 3$.
} 
rather the chance super-position of emission lines into the broad-band filters. For CDF-S 202 , the $R$ band contains both Ly $\alpha$ and CIV, while the $K s$ band contains the weaker $\mathrm{H} \beta, \mathrm{H} \delta$ and HeII. Since the CDF-S 202 optical continuum is not detected, we used the NGC 1068 template redshifted to $\mathrm{z}=3.7$ to verify the effect of line subtraction to the optical-infrared colors, assuming that the NGC 1068 spectrum is identical to that of CDF-S 202. We found that $R-K s=3.92$ when lines are subtracted, which is comparable with the Lehmann et al. findings.

Both observed and corrected (i.e. line subtracted) magnitudes are shown in Fig. 6, along with the X-ray spectrum and the radio upper limit $\left(F_{\nu}<100 \mu J y\right.$, where $F_{\nu}$ is the flux density at $20 \mathrm{~cm}$ ) derived from VLA observations (K.Kellerman, private communication). To put our data into perspective, they have been shifted to the rest frame and compared with the SED the nearby galaxy NGC 6240 (Hasinger 2001), which has no AGN signatures in its optical spectrum but shows clearly a buried AGN emerging at higher X-ray energies Vignati et al. (1999).

The inferred metallicity for this object is $\gtrsim Z_{\odot}$ typical of high redshift AGN but on the high side. In fact, looking at our diagnostic diagram, CDF-S 202 has a higher metallicity than any of the high-z radio galaxies of Vernet et al. (2001); Hamann \& Ferland (1993). Indeed, it has been found that there is a metallicity evolution such that for redshift $z>3$ radio galaxies, the metallicity is $<2 \times Z_{\odot}$ ( (de Breuck et al. 2000). This relatively high metallicity is consistent with a high star formation rate during these early epochs as the bulge and central black hole are formed.

Assume now that the $F e K \alpha$ line arises in the obscuring cloud. We will now estimate various quantities of the obscuring region using either standard parameters or, where possible, parameters inferred from our data. To simplify the calculation we assume that the obscuring cloud is spherical. From other studies (Gilli et al. 2001) we find that the Type 2 to Type 1 ratio is $\sim 10$ at high redshift and therefore it is a reasonable assumption that the obscuring torus is an almost totally enclosing cloud.

We define the ionization parameter, $\mathrm{U}$, in the usual manner as

$$
U=\frac{L_{X}}{n r^{2}}
$$

in an obscuring material of density, $n$, at distance, $r$ from the source. For standard values $\mathrm{U}=10^{2} \mathrm{erg} \mathrm{cm} \mathrm{s}^{-1}$ (Matt et al. 1996).

For the inferred absorbing column, $\mathrm{N}$, of the spherical uniform cloud, we find 


$$
N=n r
$$

For a typical inferred absorbing column we use of $N=10^{24.5} \mathrm{~cm}^{-2}$ that is consistent with canonical estimates expected for Type 2 QSOs and also with our inferred column densities in Section 4.

It follows directly that the radius of the cloud is

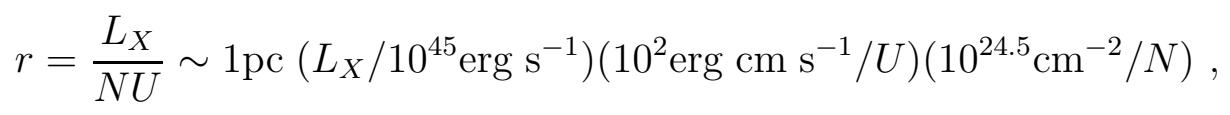

and the density of the cloud is

$$
n=\frac{N^{2} U}{L_{X}} .
$$

The mass estimate of the obscuring cloud, $M_{\text {cloud }}$, is

$$
M_{\text {cloud }}=\left(\frac{4 \pi}{3}\right) \mu m_{H} n r^{3}=\left(\frac{4 \pi}{3}\right) \mu m_{H}\left(\frac{L_{x}^{2}}{N U^{2}}\right)
$$

where $\mu$ is the mean molecular weight of the obscuring material. We then estimate the mass in the obscuring region $M_{\text {cloud }}$, to be

$$
M_{\text {cloud }} \sim 1 \times 10^{5} M_{\odot}\left(L_{X} / 10^{45} \mathrm{erg} \mathrm{s}^{-1}\right)\left(10^{2} \mathrm{erg} \mathrm{cm} \mathrm{s}^{-1} / U\right)\left(10^{24.5} \mathrm{~cm}^{-2} / N_{H}\right)
$$

We now assume that the velocity dispersion outside the obscuring cloud can still be dominated by influence of the black hole. This will not always be the case but here the numbers are sufficiently interesting and consistent to analyze the problem further. Then, the velocity dispersion, $\sigma_{\text {cloud }}$, just outside the obscuring region is given by

$$
\sigma_{\text {cloud }}^{2}=\left(\frac{G M_{B H}}{r}\right)
$$

where $M_{B H}$ is the mass of the black hole. It follows directly that

$$
\sigma_{\text {cloud }}^{2}=\left(\frac{G M_{B H}}{L_{X}}\right)(N U)
$$


We further rewrite the black hole mass in terms of the Eddington luminosity given by

$$
M_{B H}=\left(\frac{\sigma_{T}}{4 \pi G c m_{H}}\right) L_{E D D}
$$

and then

$$
\sigma_{\text {cloud }}^{2}=\left(\frac{\sigma_{T}}{4 \pi c m_{H}}\right)\left(\frac{L_{E D D}}{L_{X}}\right)(N U)
$$

Now we can estimate the velocity dispersion, just outside the obscuring region is

$$
\left.\sigma_{\text {cloud }} \lesssim 2500 \mathrm{kms}^{-1}\left[L / L_{E D D}\right] / 10^{-2}\right)^{1 / 2}\left(U / 10^{2} \mathrm{ergcms}^{-1}\right)^{1 / 2}\left(N / 10^{24.5} \mathrm{~cm}^{-2}\right)^{1 / 2}
$$

We have assumed here that the black hole is generating X-rays with an efficiency of $1 \%$ relative to the Eddington luminosity which we justify as follows. As discussed by Elvis et al. (1994) the ratio of X-ray luminosity to bolometric luminosity, $L_{b o l}$, is $10 \%$. Here we assume $L_{b o l} / L_{E D D}$ is also $10 \%$. The inferred mass of the black hole is then

$$
M_{B H} \sim 8 \times 10^{8} M_{\odot}\left(10^{-2} /\left[L_{X} / L_{E D D}\right]\right)\left(L_{X} / 10^{45} \mathrm{ergs}^{-1}\right)
$$

This explains the rather large width of the narrow lines that originate outside the obscuring region. That such a dense cloud closely covers the nucleus in high redshift Type 2 objects is to be expected since in the galaxy formation process large amounts of gas are present in the bulge-forming black-hole-growing epoch. The encapsulation of the nucleus probably increases with redshift.

We now discuss our analysis and analyze the energetics of the line emission. Following the standard scenario,we assume that:(1) the ratio of UV to X-ray luminosity is 10 (Elvis et al. 1994);(2) 10\% of the UV continuum escapes, consistent with the ratio of Type 2/Type $1 \sim 10$ at high redshift; (3) 10\% of the continuum flux is re-radiated isotropically into lines and (4) the covering factor of the narrow line region is $10 \%$. We find that the line flux should be $10^{-2} L_{X}$. The actual line flux is $L_{\text {line }} \sim 2 \times 10^{43} \mathrm{erg} \mathrm{s}^{-1}$ roughly consistent with the above model. In a similar calculation we use the observationally derived relation $L_{\text {Lyx }} \sim 10^{-3} L_{b o l}$ (Vanden Berk et al. 2001, T. Heckman, private communication) where $L_{b o l}$ is the bolometric luminosity of the QSO and $L_{L y \alpha}$ is the narrow line luminosity in $L y \alpha$. We then find that CDF-S 202 has an inferred luminosity from the Ly $\alpha$ line of $L_{b o l}=2 \times 10^{46} \mathrm{erg}$ $\mathrm{s}^{-1}$. In addition, the X-ray luminosity inferred from the line strength and the observationally 
determined value $\left(L_{x} / L_{b o l}=0.1\right.$ (Elvis et al. 1994) is $L_{x}=2 \times 10^{45} \mathrm{erg} \mathrm{s}^{-1}$. This constructs a consistent picture of CDF-S as an enshrouded Type2 QSO.

In general for observations to support such an analysis of Type 2 QSOs physics as given above we would like to study in the future a significantly lower redshift sample of X-ray selected QSO 2 to attempt to infer the depth of the potential well via the rotation curve and the central stellar velocity dispersion using, say, STIS observations. Imaging observations of such objects in CDF-S with HST can give important morphological constraints (see Schreier et al. 2001) and possibly an estimate of the bulge mass which can also be used to estimate the black hole mass (Magorrian et al. 1998; Gebhardt et al. 2000). In addition, deep Chandra and XMM observations of high-redshift Narrow Line Radio Galaxies (NLRG) can give insight into the physics of Type 2 QSOs. In unified models NLRG are the radio loud equivalent of Type 2 QSOs. It has been proposed by Ridgeway et al. (2001) that a few percent of the Lyman-break galaxy population (Steidel et al. 1999) may be Type 2 QSOs. X-ray studies of a well-selected subsample of these Lyman-break systems are also indicated.

It is expected from models that the radiation absorbed in the cold obscuring torus to be re-radiated in the far infra-red. IRAS $09014+4109$ is such an object. Interestingly, it is the only hyperluminous infrared galaxy to be detected as an obscured AGN ( Iwasawa et al. 2001). Deep IR imaging and spectroscopy are essential to constrain the contribution of QSO 2s to the IR background. Current thinking is that the AGN contribution may approach that due to star formation if sufficient Type 2 QSOs contribute. For these powerful buried QSOs at high redshift the Type2/Type1 ratio is unknown but may continue to increase with redshift giving a major contribution to the IR background. In a related issue these Type2 QSOs may be powerful submillimeter sources. We already know that a fraction of optically identified SCUBA sources are AGN (Barger et al. 1999; Smail et al. 1999) although the bulk of the sub-mm population is not likely to be powered by AGN, as suggested by the non-detections of SCUBA sources with Chandra (Hornschemeier et al. 2000). However, we must await the Atacama Large Millimeter Array (ALMA) to study them in detail in the CDF-S.

Depending on the assumed cosmology and X-ray transmission or reflection scenario, the intrinsic 2-10 keV rest frame luminosity of CDF-S 202 varies from $\sim 3 \times 10^{44}$ to $\sim 3 \times 10^{45}$ $\mathrm{erg} \mathrm{s}^{-1}$. Even assuming the lowest luminosity value, CDF-S 202 is a powerful obscured AGN, beyond the knee, or break in the slope, of the AGN X-ray luminosity function at $L_{*, X}=10^{43.7} \mathrm{ergs}^{-1}$ (Miyaji et al. 2000). Above this break luminosity, $L_{*, X}$ the X-ray selected AGNs are QSOs. Let us now discuss the implications of CDF-S 202 for the origin of the Xray background (Comastri et al. 1995). Based on the population synthesis models described 
by Gilli et al. $(2001)^{11}$, obscured AGN with $L_{2-10}>3 \times 10^{44} \mathrm{erg} \mathrm{s}^{-1}$ and $N_{\mathrm{H}}>10^{22} \mathrm{~cm}^{-2}$ should make up a significant part $(\sim 30 \%)$ of the hard X-ray background. About $70 \%$ of them should be observed at $z=1-3$, while $15 \%$ is expected at higher redshifts, assuming the AGN space density to decrease above $z \sim 3$ as found in optical and radio surveys (Schmidt, Schneider \& Gunn 1995; Fan et al. 2001; Shaver et al. 1999). On the same assumption we expect 8 obscured QSO with intrinsic luminosity $L_{2-10}>3 \times 10^{44} \mathrm{erg} \mathrm{s}^{-1}, N_{\mathrm{H}}>10^{22} \mathrm{~cm}^{-2}$ and $z>3$ to be observed in the 1 Msec CDF-S. We will have to await further analysis of these objects in CDF-S to understand the contribution of the QSO 2 population relative to the other sources that make up the X-ray background.

\section{Conclusions and Future Work}

We conclude that in the Chandra Deep Field South 1Msec exposure we have found a QSO 2 at redshift 3.700 (CDF-S 202). There is no generally accepted definition of a Type 2 QSO. We suggest that CDF-S 202 defines the class well by optical spectrum, X-ray spectrum and X-ray luminosity. Its luminosity $\left(10^{45 \pm 0.5} \mathrm{erg} \mathrm{s}^{-1}\right.$ in the $2-10 \mathrm{keV}$ band), X-ray spectrum, narrow permitted lines $\left(\sim 1500 \mathrm{~km} \mathrm{~s}^{-1}\right)$, and undetected continuum (to a limit of $\sim 3 \times 10^{-19}$ erg $\mathrm{cm}^{-2} \mathrm{~s}^{-1} \AA^{-1}$ ) make it a classic case. There are several other candidates in the field that have hard spectra and high redshift. Clearly, in Type 2 QSOs the $F e K \alpha$ feature can be used in conjunction with deep optical and IR faint object spectroscopy to determine redshifts. CDF-S 202 has the highest redshift FeK $\alpha$ line spectrum yet observed (Reeves et al. 2001).

We have discussed in the paper the implications for the X-ray background, the infrared background, the origin of the UV continuum in Type 2 objects and the relation of the growth of black holes to bulge and galaxy formation. Currently we aim to both increase the sample and to observe CDF-S 202 further with: IR spectroscopy and imaging, HST, millimeter facilities and XMM.

C.N. thanks Tim Heckman, Julian Krolik, David Neufeld, David Strickland and Tahir Yaqoob for stimulating discussions. G. Hasinger acknowledges support under DLR grant 50 OR 9908 O. R. Giacconi and C. Norman gratefully acknowledge support under NASA grant NAG-8-1527 and NAG-8-1133.

\footnotetext{
${ }^{11}$ Gilli et al. (2001) adopted $H_{0}=50 \mathrm{~km} / \mathrm{s} / \mathrm{Mpc}, \Omega_{m}=1$ and $\Omega_{\lambda}=0$. Here we quote their predictions correcting for the different cosmology adopted in this paper.
} 


\section{REFERENCES}

Almaini, O., et al. 1995, MNRAS, 277, L31.

Antonucci, R. 1993 ARA\&A, 31, 473

Barger, A.J., et al. 1999, AJ, 117, 2656

Comastri, A., Setti, G., Zamorani, G., \& Hasinger, G. 1995, A\&A, 296, 1

Contini, M. \& Viegas, S. M. 2000, ApJ, 535, 72

de Breuck,C., van Breugel, W., Rottgering, H.J, Miley, G \& Best, P 2000 A\&A362, 519

Elvis, M., Wilkes, B., McDowell, J., Green, R., Bechtold, J., Willner, S., Oey, S., Polomski, E., \& Cutri, R. 1994, ApJS, 95, 1

Fabian, A.C., Smail, I., Iwasawa, K., Allen, S.W., Blain, A.W., Crawford, C.W., Ettori, S., Ivison, R.J., Johnstone, R.M., Kneib, J.P. \& Wilman, R.J. 2000, MNRAS, 315, L8

Fan, X., Strauss, M.A., Schneider, D.P., et al. 2001, AJ, 121, 54

Forster, K., Green, P. J., Aldcroft, T. L., Vestergaard, M., Foltz, C. B., \& Hewett, P. C. 2001, ApJS, 134, 35

Franceschini, A., Bassani, L., Cappi, M., Granato, G.L., Malaguti, G., Palazzi, E., \& Persic, M. 2000, A\&A, 353, 910

Francis, P., Hewett, P., Foltz, C., Chaffee, F., Weymann, R., \& Morris, S. 1991, ApJ, 373, 465

Gebhardt et al. 2000, ApJ, 539, L13

Georgantopoulos, et al. 1999, MNRAS, 305, 125

Ghisellini, G., Haardt, F., \& Matt, G. 1994, MNRAS, 267, 743

Giacconi, R., Rosati, P., Tozzi, P., Hasinger, G., Norman, C., Bergeron, J., Borgani, S., Gilli, R., Gilmozzi, R. \& Zheng, W. 2001, ApJ, 551, 624

Giacconi, R., et al. 2001b, ApJsubmitted

Gilli, R., Salvati, M., \& Hasinger, G. 2000 A\&A, 366, 407

Goodrich, R. W. 1989, ApJ, 342, 224 
Granato, G., Danese, L. \& Franceschini, A. 1997 ApJ486, 147

Heckman, T., Krolik, J., Meurer, G., Calzetti, D., Kinney, A., Koratkar, A., Leitherer, C., Robert, C., \& Wilson, A. 1995, ApJ, 452, 549.

Halpern, J.L., Turner, T.J. \& George, I.M. 1999, MNRAS, 307, L47

Hamann, F. \& Ferland, G. 1993, ApJ, 418, 11

Hasinger, G. 2001, in the proceedings of "ISO Surveys of a Dusty Universe", Lemke D., Stickel M., Wilke K. eds., Springer, in press [astro-ph/0001360]

Hornschemeier, A., et al. 2000, ApJ, 541, 49

Iwasawa, K., Fabian, A.C. \& Ettori, S. 2001, MNRAS, 321, L15

Kellerman, K.I., Fomalont, E.B., Rosati, P., \& Shaver, P. 2001 in the proceedings of ESO Workshop on Deep Fields, Garching, 2001, in press

Khachikian, E. Ye \& Weedman, D. W. 1971, Astrofizika, 7, 389

Khachikian, E. Ye \& Weedman, D. W. 1974, ApJ, 192, 581

Kinney, A. L., Bohlin, R. C., Calzetti, D., Panagia, N., \& Wyse, R. F. G. 1993, ApJS, 86, 5

Kleinmann, S. G., Hamilton, D., Keel, W. C., Wynn-Williams, C. G., Eales, S. A., Becklin, E. E., \& Kuntz, K. D. 1988, ApJ, 328, 161

Krolik, J.H., Madau, P., \& Życki, P.T. 1994, ApJ, 420, L57

Lehmann, I., Hasinger, G., Schmidt, M., Giacconi, R., Trümper, J., Zamorani, G., Gunn, J. E., Pozzetti, L., Schneider, D. P., Stanke, T., Szokoly, G., Thompson, D., Wilson, G. 2001, A\&A, 371, 833

Magorrian, J., et al. 1998, AJ, 481, 673

Maiolino, R., Salvati, M., Bassani, L., Dadina, M., Della Ceca, R., Matt, G., Risaliti, G., Zamorani, G. 1998, A\&A, 338, 781

Matt, G., Fabian, A.C. and Ross, R. 1996, MNRAS, 278, 1111.

McCarthy, P. 1993, ARA\&A, 32, 639

Mitsch, W., Rupprecht , G., Seifert, W., Nicklas, H., \& Kiesewetter, S. 1994, in Instrumentation in Astronomy (Proc. SPIE Vol 2198), ed. D. L. Crawford, E. R. Craine, 317 
Miyaji, T., Hasinger, G. \& Schmidt, M. 2000, A\&A, 353, 25

Ohta, K., Yamada, Y., Nakashini, K., Ogasaka, Y. Kii, T. \& Hayashida, K. 1996 ApJ, 458, L57

Peterson, B. M. 1997, An Introduction to Active Galactic Nuclei (Cambridge: Cambridge Univ. Press)

Reeves, J.N., Turner, M.J.L., 2000, MNRAS, 316, 234

Reeves, J.N., Turner, M.J.L., Bennie, P.J., Pounds, K.A., Short, A., O’Brien, P.T., Boller, Th., Kuster, M., Tiengo, A., et al. 2001 A\&A, 365, L116

Ridgeway, S.E., Heckman, T.M, Calzetti, D., \& Lehnert, M. 2001 ApJ, in press

Risaliti G., Maiolino R., Salvati M., 1999, ApJ, 522, 157

Rosati, P., et al. 2001, ApJ in press, [astro-ph/0110452 ]

Efstathiou, A. \& Rowan-Robinson, M. 1995 MNRAS273, 649

Schmidt, M., Schneider, D.P., Gunn, J.E., 1995, AJ, 110, 68

Schreier, E.J., Koekemoer, A.M., Grogin, N.A., Giacconi, R., Gilli, R., Kewley, L., Norman, C., Hasinger, G., Rosati, P., Marconi, A., Salvati, M., Tozzi, P.et al. 2001, ApJ, 560, 127

Shaver, P.A., Hook, I.M., Jackson, C.A., et al. 1999, In: Carilli, C., Radford S., Menten K., Langston G., (eds.) Highly Redshifted Radio Lines. ASP Conf. Series Vol. 156, p.163 [astro-ph/9801211]

Smail, I., Ivison, R.J., Blain, A.W., \& Kneib, J.-P., in After the Dark Ages: When Galaxies were Young, eds. Holt, S., Smith, E.P, (AIP) 1999, p.312

Steidel, C., Adelberger, K., Giavalisco, M., Dickinson, M., \& Pettini, M. 1999 ApJ, 519, 1

Stern, D., et al. 2001, A\&A, in press

Tacconi, L.J., Genzel, R., Tecza, M., Gallimore, J.F., Downes, D. \& Scoville, N.Z. 1999, ApJ, 524, 732

Tozzi, P., Rosati, P., Nonino, M., Bergeron, J., Borgani, S., Gilli, R., Gilmozzi, G., Hasinger, G., Grogin, N., Kellerman, K., Kewley, L., Koekemoer, A., Norman, C., Schreier, E., Shaver, P., Wang, J.X., Zheng, W., Zirm, A. \& Giacconi, R. ApJ, in press 
Tran, H.D., Cohen, M.H., \& Villar-Martin, M. 2000 ApJ120, 562

Vanden Berk, D. E. et al., 2001, AJ, 122, 549

Vernet, J., Fosbury, R. A. E., Villar-Martin, M., Cohen, M. H., Cimatti, A., di Serego Alighieri, S. \& Goodrich, R. W. 2001, A\&A, 366, 7

Vignati, P., Molendi, S., Matt, G., Guainazzi, M., Antonelli, L.A., Bassani, L., Brandt, W.N., Fabian, A.C., Iwasawa, K., Maiolino, R., Malaguti, G., Marconi, A., \& Perola, G.C. 1999 A\&A, 349, L5

Villar-Martin, M., Fosbury, R. A. E., Binette, L., Tadhunter, C. N., \& Rocca-Volmerange, B. 1999, A\&A, 351, 47

Weedman, D. W. 1970, ApJ, 159, 405

Weedman, D. W. 1973, ApJ, 183, 29

Yaqoob, T., George, I.M., Nandra, K., Turner, T.J., Zobair, S., \& Serlemitsos, P.J. 1999 ApJ, 525, 19 

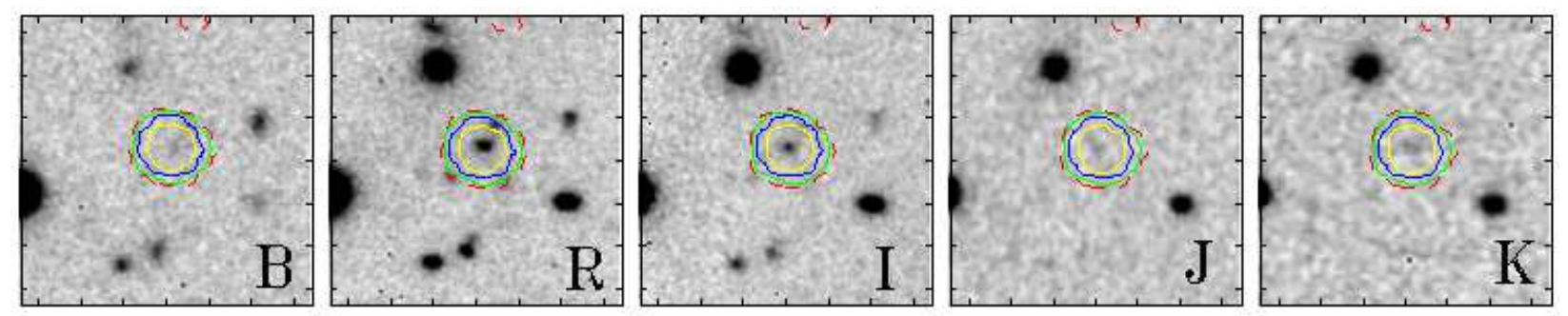

Fig. 1.- Optical and near IR images with the X-ray brightness contours overlaid. Optical and near IR images are $20^{\prime \prime}$ across; the X-ray contours correspond to 3,5,10 and $20 \sigma$ levels above the background for the $2-7 \mathrm{keV}$ image 


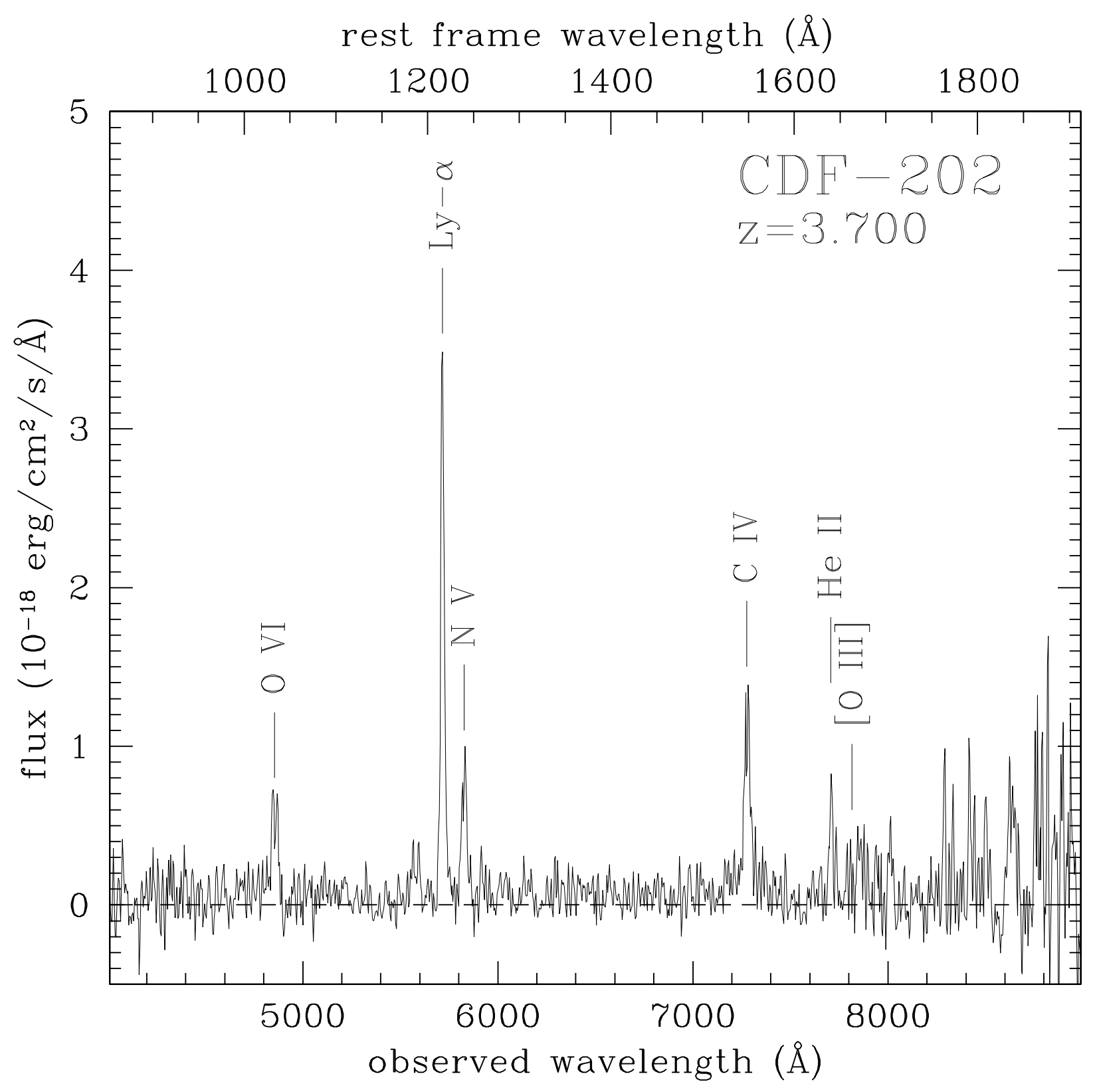

Fig. 2.- Low resolution optical spectrum of CDF-S 202 obtained with VLT UT1 FORS1. The emission features that the redshift determination is based on are marked. Wavelength calibration inaccuracies limit the accuracy of redshift determination to \pm 0.005 . Flux calibration of emission lines is good to $5 \%$ in the range displayed. Data were not corrected for slit loss, which we estimate to be around $30 \%$ and nearly achromatic. 


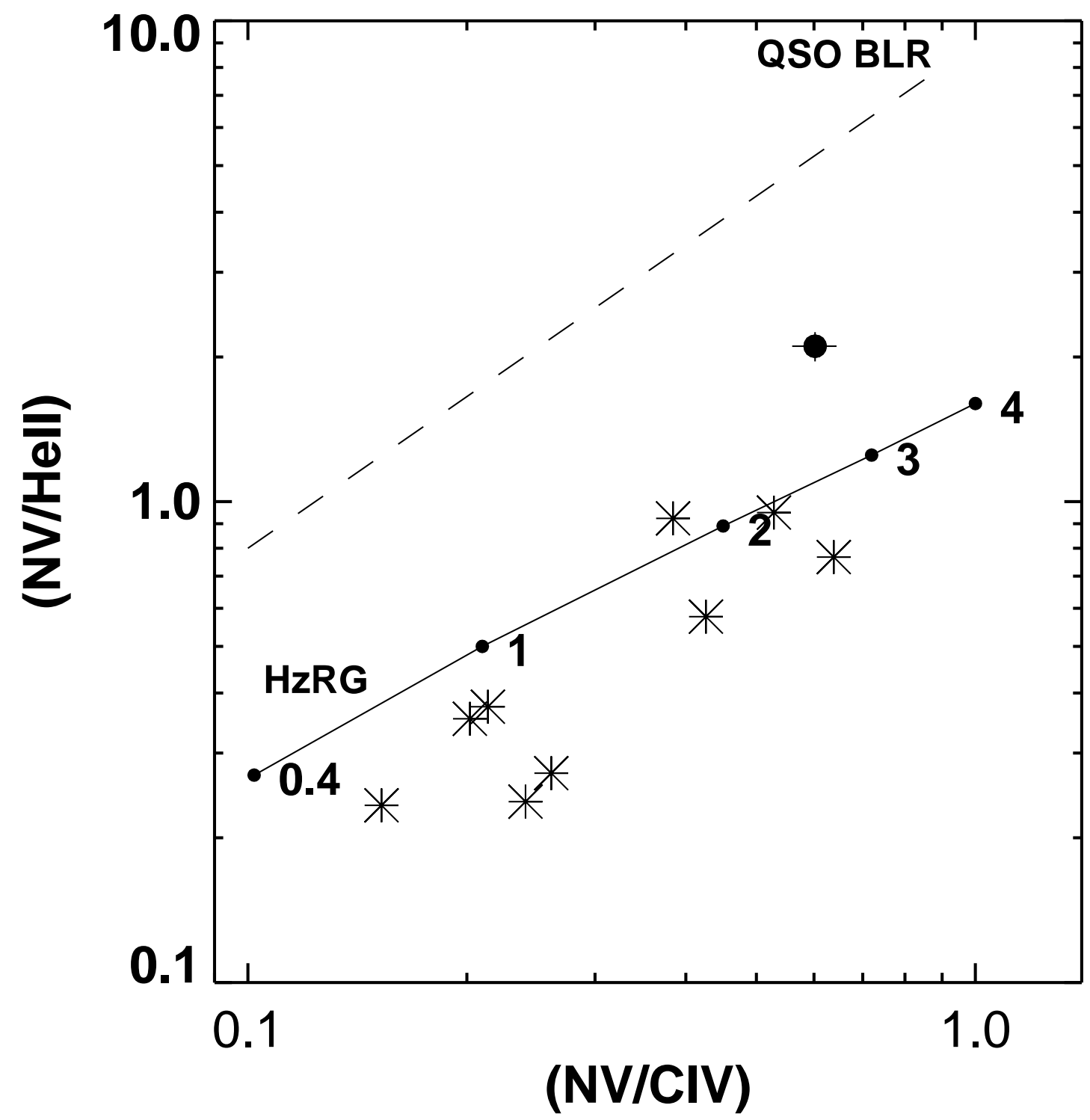

Fig. 3.- The N V/He II vs. N V/C IV diagram as plotted by Hamann \& Ferland (1993); Vernet et al. (2001). The solid line gives the locus of the best-fit power-law photoionization models for metallicities ranging from $0.4-4 Z_{\odot}$.THe BLR model for QSOs (Hamann \& Ferland 1993) is given by the dashed line. NLRG are shown as asterisks and CFD-S 202 as a filled circle. CFD-S 202 appears to have high metallicity. 


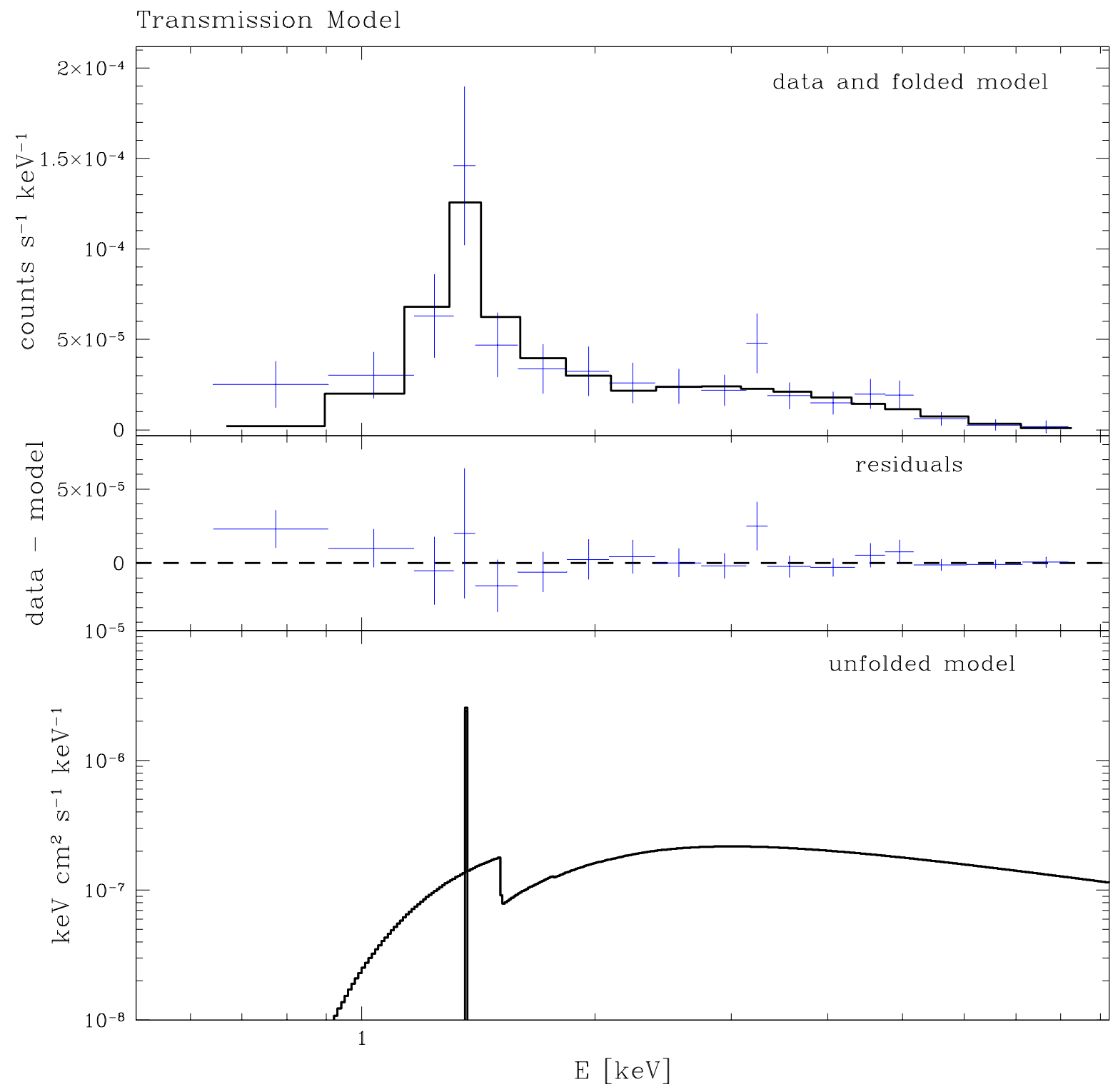

Fig. 4.- Top panels: X-ray Spectrum of CDF-S 202 with Transmission Fit and residuals. Bottom panel: the unfolded best-fit model. 


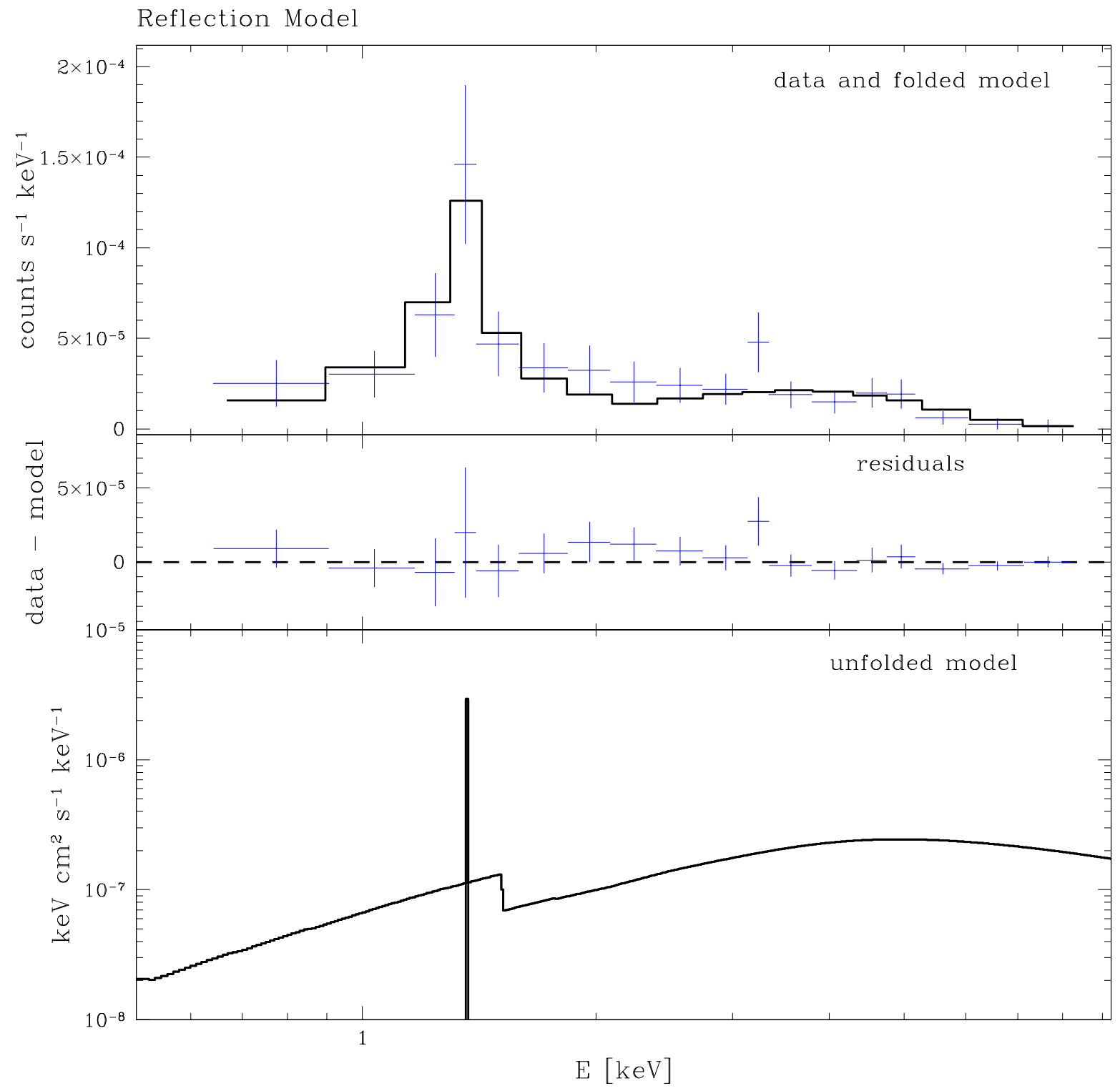

Fig. 5.- Top panels: X-ray Spectrum of CDF-S 202 with Reflection Fit and residuals. Bottom panel: the unfolded best-fit model. 


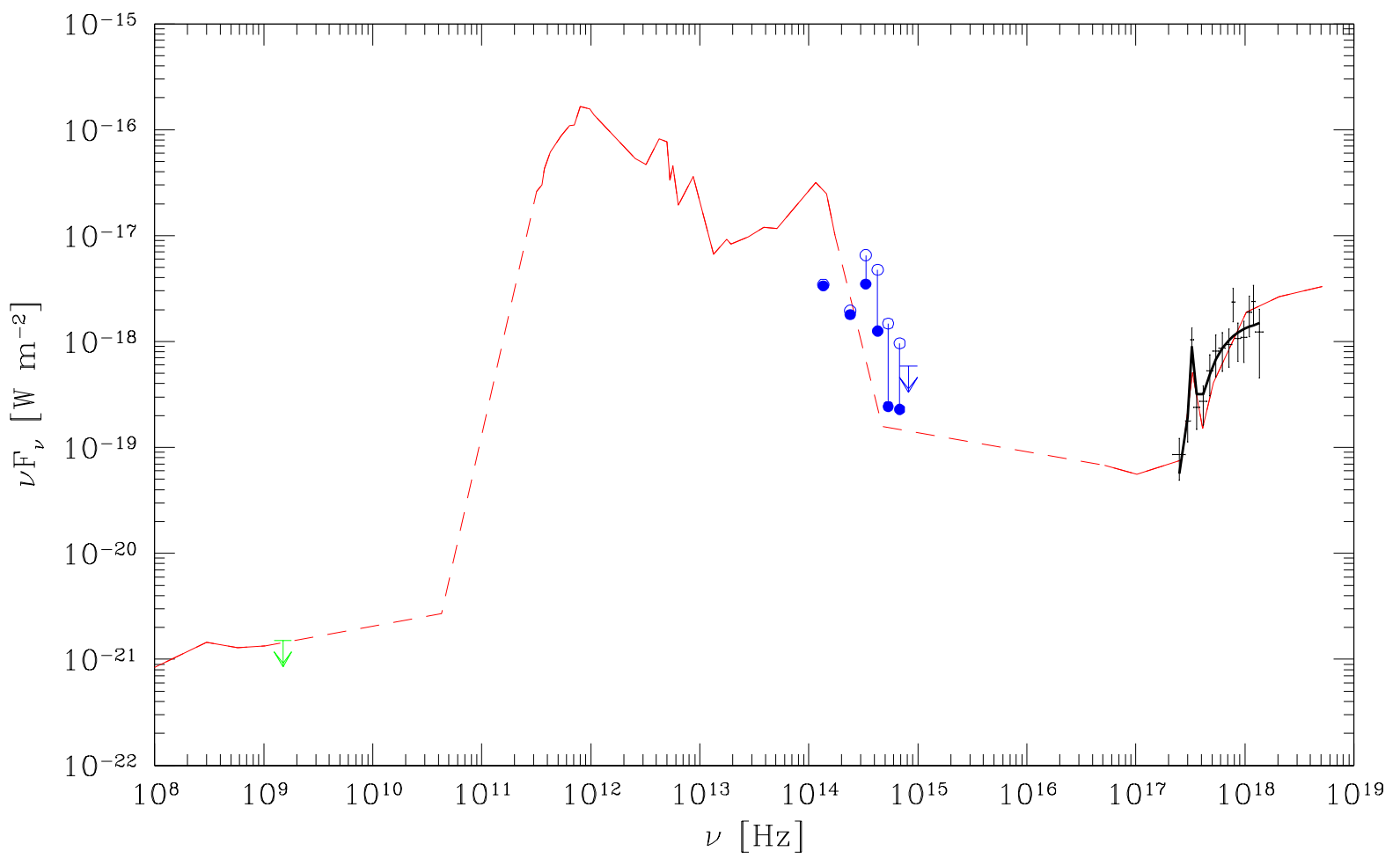

Fig. 6.- Detections and upper limits for CDF-S 202 plotted over the SED for the local Type 2 template, NGC 6240 from Hasinger (2001) (solid line; dashed line where the template is extrapolated). The CDF-S 202 data have been shifted to the rest-frame. The NGC 6240 SED has been normalized to the X-ray spectrum of CDF-S 202. Open circles and filled circles refer to observed magnitudes and to line subtracted magnitudes, respectively (see the Discussion). 
Table 1. CDF-S 202 Source Properties

\begin{tabular}{|c|c|c|c|c|c|c|c|}
\hline \multicolumn{8}{|l|}{ X-ray Data } \\
\hline Model & Transmission & Reflection & & & & & \\
\hline$\Gamma$ (fixed) & 1.8 & 1.8 & & & & & \\
\hline$N_{H}\left(10^{23} \mathrm{~cm}^{-2}\right)$ & $7.9_{-2.3}^{+3.5}$ & $\geq 10^{2}$ & & & & & \\
\hline $\mathrm{EW}_{\mathrm{Fe} \mathrm{K} \alpha}^{a}($ rest-frame eV) & $823_{-823}^{+1694}$ & $1186_{-922}^{+1195}$ & & & & & \\
\hline$F_{0.5-2}^{b}\left(10^{-16} \mathrm{erg} \mathrm{s}^{-1} \mathrm{~cm}^{-2}\right)$ & $2.21 \pm 0.33$ & $2.25 \pm 0.33$ & & & & & \\
\hline$F_{2-10}^{b}\left(10^{-16} \mathrm{erg} \mathrm{s}^{-1} \mathrm{~cm}^{-2}\right)$ & $20.6 \pm 3.0$ & $25.8 \pm 3.5$ & & & & & \\
\hline \multicolumn{8}{|l|}{ Optical Imaging } \\
\hline Band & $U$ & $B$ & $V$ & $R$ & $I$ & $J$ & $K s$ \\
\hline Vega magnitudes & $>26$ & $26.2 \pm 0.3$ & 25.27 & 23.53 & $22.65 \pm 0.1$ & $23.28 \pm 0.15$ & $20.97 \pm 0.11$ \\
\hline \multicolumn{8}{|c|}{ Optical Spectroscopy (rest frame) } \\
\hline & Ly $\alpha$ & NV & CIV & HeII & & & \\
\hline $\mathrm{F}\left(10^{-18} \mathrm{erg} \mathrm{s}^{-1} \mathrm{~cm}^{-2}\right)^{c}$ & 16.4 & 5.9 & 9.9 & 2.8 & & & \\
\hline FWHM $(\AA)$ & $<5$ & $<7$ & $<9$ & $<4$ & & & \\
\hline Velocity Width $\left(\mathrm{km} \mathrm{s}^{-1}\right)$ & $<1130$ & $<1680$ & $<1680$ & $<680$ & & & \\
\hline \multicolumn{8}{|l|}{ IRAS Imaging } \\
\hline Band & $S_{12 \mu}$ & $S_{25 \mu}$ & $S_{60 \mu}$ & $S_{100 \mu}$ & & & \\
\hline$F_{\nu}(\mathrm{Jy})$ & $<0.2$ & $<0.2$ & $<0.2$ & $<1.0$ & & & \\
\hline \multicolumn{8}{|l|}{ VLA $20 \mathrm{~cm}$ Imaging } \\
\hline$F_{\nu}(\mu \mathrm{Jy})$ & $<100$ & & & & & & \\
\hline
\end{tabular}

